\title{
Pantoea Species Bacteremia in a Child With Sickle Cell Disease: Looking for a Culprit
}

\author{
Marisa I. Oliveira, MD, * Sara Batalha, MD, ${ }^{*}$ Catarina Gouveia, MD, $\dagger$ Raquel \\ Maia, MD,* and Paula Kjöllerstrom, MD*
}

\begin{abstract}
Summary: Pantoea agglomerans has been classically associated with cellulitis or synovitis secondary to penetrating trauma by vegetation. It is an infrequent cause of systemic infections. We describe the case of a 5-year-old girl with sickle cell disease with $P$. agglomerans bacteremia and review its potential causes.
\end{abstract}

Key Words: Pantoea agglomerans, sickle cell disease, blood products, pica

(J Pediatr Hematol Oncol 2017;39:e307-e308)

\section{BACKGROUND}

Pantoea agglomerans, formerly known as Enterobacter agglomerans, is a gram-negative nonencapsulated aerobic bacillus member of the Enterobacteriaceae family. ${ }^{1}$ It is a ubiquitous microorganism that can be found in soil, plants, water, feces, and foodstuffs $\mathrm{s}^{1,2}$ and an opportunistic pathogen that very rarely causes disease in healthy individuals. 1,3 Immunodeficiency is an established risk factor. ${ }^{4}$ Most infections are classically cellulitis, arthritis, or synovitis secondary to penetrating trauma by vegetation, ${ }^{1-4}$ but $P$. agglomerans nosocomial bacteremia is also well-described. It is usually associated with indwelling catheters, ${ }^{5}$ contaminated intravenous (IV) fluids, ${ }^{2}$ and blood products. ${ }^{6}$ Cases of liver abscess $^{7}$ and endophtalmitis ${ }^{8}$ have been documented. Bacterial translocation from the gut during gastroenteritis has also been postulated. ${ }^{9}$

\section{OBSERVATION}

A 5-year-old girl with a medical history of prematurity, failure to thrive, and homozygous SS sickle cell disease (under amoxicillin prophylaxis) with prior admission for acute chest syndrome presented to the emergency department with a history of high-grade fever for the past three days $\left(39^{\circ} \mathrm{C}\right)$ and productive cough. Other than previously known pallor, mild jaundice, and systolic murmur, her physical examination was unremarkable. Laboratory values showed anemia with a hemoglobin $(\mathrm{Hb})$ of $7.8 \mathrm{~g} / \mathrm{dL}$, leukocytosis $(36,300 / \mu \mathrm{L}, 78 \%$ neutrophils), thrombocytosis $(489,000 / \mu \mathrm{L})$, and an increased C-reactive protein (CRP) of $45.2 \mathrm{mg} / \mathrm{L}$ (reference value $<5.0 \mathrm{mg} / \mathrm{L}$ ). The chest $\mathrm{x}$-ray performed was normal. She was admitted with the diagnosis of fever of unknown origin and was started on IV cefotaxime (100 mg per $\mathrm{kg}$ per day) according to the protocol.

Received for publication April 24, 2016; accepted February 6, 2017. From the ${ }^{*}$ Hematology Unit; and $\dagger$ Infectious Diseases Unit, Hospital D. Estefânia, Centro Hospitalar de Lisboa, Central, EPE, Lisbon, Portugal.

The authors declare no conflict of interest.

Reprints: Marisa I. Oliveira, MD, Hospital D. Estefânia, Rua Jacinta Marto, Lisbon 1169-045, Portugal (e-mail: marisaioliveira@gmail.com). Copyright (C) 2017 Wolters Kluwer Health, Inc. All rights reserved.
After 24 hours on IV antibiotics, she was afebrile. The blood culture obtained on admission was sterile. Despite clinical improvement, hospitalization was prolonged due to the development of lumbar and abdominal pain attributed to vasoocclusive crisis. On day 7, laboratory evaluation revealed significant hemolysis, with a decrease in $\mathrm{Hb}$ concentration $(7.0 \mathrm{~g} / \mathrm{dL})$, elevation of lactate dehydrogenase $(485 \mathrm{U} / \mathrm{L}$; reference value $110-295 \mathrm{U} / \mathrm{L}$ ) and elevation of total bilirrubin $(1,59 \mathrm{mg} / \mathrm{dL}$; reference value $0.3-1.2 \mathrm{mg} / \mathrm{dL})$, increased leukocyte count $(23,700 / \mu \mathrm{L})$, and CRP $(26,6 \mathrm{mg} / \mathrm{L})$. The patient received a unit of packed red blood cell transfusion. During the transfusion, she developed high fever $\left(39^{\circ} \mathrm{C}\right)$ that persisted for 4 days (every $8 \mathrm{~h}, 38,5^{\circ} \mathrm{C}$ ). Lab testing was remarkable for worsening leukocytosis $(38,900 / \mu \mathrm{L})$ and CRP $(76,8 \mathrm{mg} / \mathrm{L})$. Antibiotic therapy was broadened with the addition of IV metronidazole and vancomycin. Blood samples were drawn into aerobic and anaerobic blood culture bottles (BacT/Alert FA; bioMerieux, Mercy l'Etoile, France). After a 12-hour incubation period (incubator BacT/Alert 3D; bioMerieux, Mercy l'Etoile, France), the aerobic blood culture was positive for $P$. agglomerans sensitive to amoxicillin/clavulanic acid, cefuroxime, and cefotaxime. For strain identification, MALDI-TOF Mass Spectrometry (Vitek MS system, bioMérieux, Marcy ÍÉtoile, France), reported to be a reliable method for the identification of the genus Pantoea, ${ }^{10}$ confirmed the strain. Therefore, molecular confirmation of $P$. agglomerans identity was not considered necessary.

No other effects of bacteremia were observed; the patient remained hemodynamically stable and did not require additional transfusional support.

The patient denied prior penetrating trauma or diarrhea and had no central venous line that could help establish an underlying cause for Pantoea bacteremia. After questioning, the mother referred pica in the 6 months before admission (ingestion of wall paint and soil).

Unfortunately, environmental samples (glucose solutions and transfused blood) cultures were not performed. There were no other Pantoea infections reported in the hospital in this period. Other reported associations were not found in this case: there was no history of gastrointestinal symptoms or lesions or recent antiacid administration. Also, cotton pledgets are not used in our institution and plants and flowers are forbidden in the interior of the hospital, ruling out these as possible contamination sources.

Four days later, the patient was afebrile and clinically well. Blood culture was repeated and sterility documented. The patient was discharged on day 17 of hospitalization, back to her usual state of health after a total course of 17 days of IV cefotaxime, 10 days of IV metronidazole and vancomycin, and was discharged on oral amoxicillin/ clavulanic (total of $10 \mathrm{~d}$ of antimicrobial therapy after positive blood culture). 


\section{DISCUSSION}

Previous reports have implicated Pantoea spp in systemic infections in immunocompromised hosts. ${ }^{11}$ Sickle cell disease patients are known to have impaired immunity beyond functional asplenia. ${ }^{12,13}$ Achkar et $\mathrm{al}^{4}$ have already reported a case of $P$. agglomerans bacteremia in a sickle cell disease patient. In addition, $P$. agglomerans bacteremia has been associated with blood type A, as was the blood type of this patient. ${ }^{14}$

Infections caused by $P$. agglomerans are usually associated with an identifiable exogenous source. ${ }^{3}$ In this case, we identified several factors that could explain Pantoea spp bacteremia.

The patient denied plant injury, but admitted ingestion of soil and wall paint six months before admission, which could explain the contamination, followed by translocation reaching the bloodstream and causing infection, a mechanism described previously. ${ }^{4,9}$

Despite pica being remarkably common in sickle cell pediatric patients ${ }^{15}$ and bacterial translocation a relatively frequent occurrence in this patient population due to focal gut mucosal ischemia, Pantoea bacteremia is not frequent in these patients, which reduces the likelihood of pica as a source in this case. Also, a positive culture that occurred while the patient was under antimicrobial therapy to which the isolate was highly sensitive also argues against that hypothesis.

The fact that a $P$. agglomerans-positive culture occurred after hospitalization and while the patient was under antibimicrobial therapy to which the isolate was highly sensitive also argues against that hypothesis. Although it is difficult to draw a definite conclusion, it is tempting to assume that this case of $P$. agglomerans bacteremia might represent nosocomial contamination, possibly related to packed red blood cell transfusion.

\section{CONCLUSIONS}

This case illustrates a very rare cause of bacteremia in sickle cell patients and the multitude of confounding factors that can be present, making the establishment of a timeline difficult. It is important to exclude nosocomial infection to prevent hospital outbreaks.

\section{REFERENCES}

1. Cruz AT, Cazacu AC, Allen CH. Pantoea agglomerans, a plant pathogen causing human disease. J Clin Microbiol. 2007;45: 1989-1992.

2. Bicudo EL, Macedo VO, Carrara MA, et al. Nosocomial outbreak of Pantoea agglomerans in a pediatric urgent care center. Braz J Infect Dis. 2007;11:281-284.

3. Tiwari S, Beriha SS. Pantoea species causing early onset neonatal sepsis: a case report. J Med Case Rep. 2015;9: 188-188.

4. Achkar Al M, Rogers JS, Muszynski MJ. Pantoea species sepsis associated with sickle cell crisis in a pregnant woman with a history of pica. Am J Case Rep. 2012;13:26-28.

5. Wong K-W. Pantoea agglomerans as a rare cause of catheterrelated infection in hemodialysis patients. $J$ Vasc Access. 2013;14:306-306.

6. Alvarez FE, Rogge KJ, Tarrand J, et al. Bacterial contamination of cellular blood components. A retrospective review at a large cancer center. Ann Clin Lab Sci. 1995;25:283-290.

7. Rodrigues A, Lima IK, Junior AK. Pantoea agglomerans liver abscess in a resident of Brazilian Amazonia. Trop Gastroenterol. 2009;30:154-155.

8. Lee NE, Chung IY, Park JM. A case of Pantoea endophthalmitis. Korean J Ophthalmol. 2010;24:318-321.

9. Cicchetti R, Iacobini M, Midulla F, et al. Pantoea agglomerans sepsis after rotavirus gastroenteritis. Pediatr Infect Dis J. 2006; 25:280-281.

10. Richter SS, Sercia L, Branda JA, et al. Identification of Enterobacteriaceae by matrix-assisted laser desorption/ionization time-of-flight mass spectrometry using the VITEK MS system. Eur J Clin Microbiol Infect Dis. 2013;32:1571-1578.

11. Liberto MC, Matera G, Puccio R, et al. Six cases of sepsis caused by Pantoea agglomerans in a teaching hospital. New Microbiol. 2009;32:119-123.

12. Battersby AJ, Knox-Macaulay HHM, Carrol ED. Susceptibility to invasive bacterial infections in children with sickle cell disease. Pediatr Blood Cancer. 2010;55:401-406.

13. Booth $\mathrm{C}$, Inusa $\mathrm{B}$, Obaro $\mathrm{SK}$. Infection in sickle cell disease: a review. Int $J$ Infect Dis. 2010;14:e2-212.

14. Cheng A, Liu C-Y, Tsai H-Y, et al. Bacteremia caused by Pantoea agglomerans at a medical center in Taiwan, 2000-2010. J Microbiol Immunol Infect. 2013;46:187-194.

15. Ivascu NS, Sarnaik S, McCrae J, et al. Characterization of pica prevalence among patients with sickle cell disease. Arch Pediatr Adolesc Med. 2001;155:1243-1247. 